\title{
Characteristics of Ga doped ZnO Thin Films Deposited by RF Magnetron Sputtering with Base Pressure
}

\author{
Deok Kyu Kim* \\ Department of Electrical Engineering, Wonkwang University, Iksan 54538, Korea
}

Received December 27, 2018; revised January 17, 2019; accepted January 31, 2019

\begin{abstract}
Ga doped $\mathrm{ZnO}$ thin films were deposited with various base pressure by RF magnetron sputtering, and their structural, optical, and electrical properties were studied. Effects of base pressure on the properties of Ga doped $\mathrm{ZnO}$ thin films were confirmed and a high quality thin film was obtained by controlling the base pressure. In our case, as the base pressure decreases, the crystal quality is improved, the surface roughness is increased, the sheet resistance is decreased and the figure of merit is increased. According to X-ray diffraction results, the decrease of sheet resistance is caused by increasing the grain size. The transmittance in visible region $(400 \sim 800 \mathrm{~nm})$ had $85 \sim 87 \%$. Therefore, the base pressure is a key parameter for obtaining high quality film.
\end{abstract}

Keywords: Ga doped ZnO, RF magnetron sputtering, Base pressure, Grain size

\section{Introduction}

As the IT industry develops rapidly, the transparent conductive oxides (TCOs) has been increasing interest. TCOs is an essential element for optoelectronic devices such as solar cells, light emitting diodes, and organic light emitting diodes [1-3]. In general, Indium tin oxide (ITOs) are widely used as TCOs due to its low resistivity and high visible transmittance. However, the toxicity and high cost of the indium in the ITO lead to the demand for new TCOs. Zinc oxide $(\mathrm{ZnO})$ system doped Group III metal elements ( $\mathrm{Al}$, In and $\mathrm{Ga}$ ) has many attentions to TCOs [46]. In especial, $\mathrm{Ga}$ doped $\mathrm{ZnO}$ (GZO) is a promising candidate due to several advantages such as less reactive and more resistant to oxidation than $\mathrm{Al}$ [7]. Many methods have been used to deposit GZO films, such as RF magnetron sputtering, pulsed laser deposition, atomic layer deposition [8-10]. From these deposition methods, RF magnetron sputtering has been investigated due to the many advantages such as low substrate temperature and large scale deposition [11-13]. In this study, GZO thin films were deposited by the RF magnetron sputtering with base pressure. We reported the structural, electrical, and optical properties of GZO thin films.

*Corresponding author

E-mail:-dkkim17@wku.ac.kr

\section{Experimental procedure}

GZO films were prepared on corning 1737 glass substrates in RF magnetron sputtering using a $\mathrm{ZnO}: \mathrm{Ga}$ ( $97: 3 \mathrm{wt} \%$ ) ceramic target at room temperature. The glass substrates were cleaned with acetone, methanol, and deionized water. The base pressure of the chamber was evacuated by rotary pump and turbo molecular pump and maintained at a pressure to $2.5 \times 10^{-6}, 6.5 \times 10^{-6}$, and $10.5 \times 10^{-6}$ mbar by using the throttle gate valve. Then, high purity $\operatorname{Ar}(40 \mathrm{sccm})$ was introduced. At the fixed sputtering power of $25 \mathrm{~W}$, the GZO films were deposited at sputtering pressure of $8 \times 10^{-3} \mathrm{mbar}$ by throttle valves. GZO films with about same thickness of $200 \mathrm{~nm}$ were deposited by controlling sputtering time. Also, the sputtering was carried out in $65 \%$ of relative humidity. The deposition conditions for GZO thin film are summarized in Table I.

The structural properties of GZO thin films were

Table I. Sputtering conditions of GZO thin films.

\begin{tabular}{cc}
\hline \hline Parameters & Conditions \\
\hline Target & ZnO:Ga (97:3) \\
Substrate & Glass (corning 1737) \\
Base Pressure [mbar] & $(2.5,6.5,10.5) \times 10^{-6}$ \\
Working Pressure [mbar] & $8 \times 10^{-3}$ \\
RF power [W] & 25 \\
Ar : $\mathrm{O}_{2}[\mathrm{sccm}]$ & 50 \\
Depo. Temperature & $\mathrm{RT}$ \\
Film Thickness [nm] & 200 \\
\hline
\end{tabular}


measured by X-ray diffraction (XRD) analysis. The surface morphology was analyzed by atomic force microscope (AFM). 4-point probe was used for sheet resistance of films. The optical properties were measured by UV/VIS/ NIR spectrophotometer for transmittance of GZO thin films.

\section{Results and discussions}

Figure 1 shows XRD patterns of GZO thin films with various base pressures. All GZO thin films show an only strong peak at $34.5^{\circ}$ which is related to the $\mathrm{ZnO}(0002)$ plane, indicating direction of the $\mathrm{c}$-axis perpendicular to the surface. The peak intensity and full width at half maximum (FWHM) of the (002) peaks for GZO films vary depending on the base pressure. With decreasing the background pressure from $10.5 \times 10^{-6}$ to $2.5 \times 10^{-6}$ mbar, the peak intensity increases from 5221 to 8935 and FWHM decreases from $0.4^{\circ}$ to $0.34^{\circ}$. These results suggest that the low base pressure leads to a high preferred orientation (c plane) and a high crystallinity of GZO thin films due to the decrease of residual impurity in the chamber. In general, residual impurity such as nitrogen and oxygen is in the chamber and interrupt the crystal growth. Therefore, the low residual impurity at $2.5 \times 10^{-6}$ mbar reduces the interruption of the GZO crystal growth, resulting in improving the crystal quality [14]. We calculated the grain size using Scherrer's formula [15]:

$$
D=\frac{0.9 \lambda}{B \cos \theta}
$$

where $\lambda$ is the $\mathrm{X}$-ray wavelength (1.5406 $\AA$ ), $\mathrm{B}$ is the FWHM and $\theta$ is the Bragg diffraction angle. The grain size of GZO thin films increases from 21 to $25 \mathrm{~nm}$ with decreasing the base pressure. From XRD results, we find that the base pressure affects the crystal quality and the microstructures of GZO thin films.

Figure 2 is AFM 3D images of GZO thin films with various base pressure. The image scale is $3 \mu \mathrm{m} \times 3 \mu \mathrm{m}$. The surface morphology of all films shows a continuous and dense hill-like surface. As the base pressure decreases from $10.5 \times 10^{-6}$ to $2.5 \times 10^{-6} \mathrm{mbar}$, the RMS roughness increases from 1.83 to $2.08 \mathrm{~nm}$. According to the results of $\mathrm{XRD}$ and AFM, this phenomenon is attributed to improve the crystal growth due to the reduction of residual impurity [16]. It is found that the RMS roughness is dependent on base pressure.

Figure 3 displays the sheet resistance (Rs) of GZO thin films with various base pressure. The sheet resistance varies depending on the base pressure. With decreasing the background pressure from $10.5 \times 10^{-6}$ to $2.5 \times 10 \times{ }^{-6} \mathrm{mbar}$, the sheet resistance decreases from 290 to $10 \Omega / \square$. The result of sheet resistance agrees with the result of XRD. That is, the decrease of sheet resistance is ascribed to the increase of grain size. Igasaki reported that the increase of

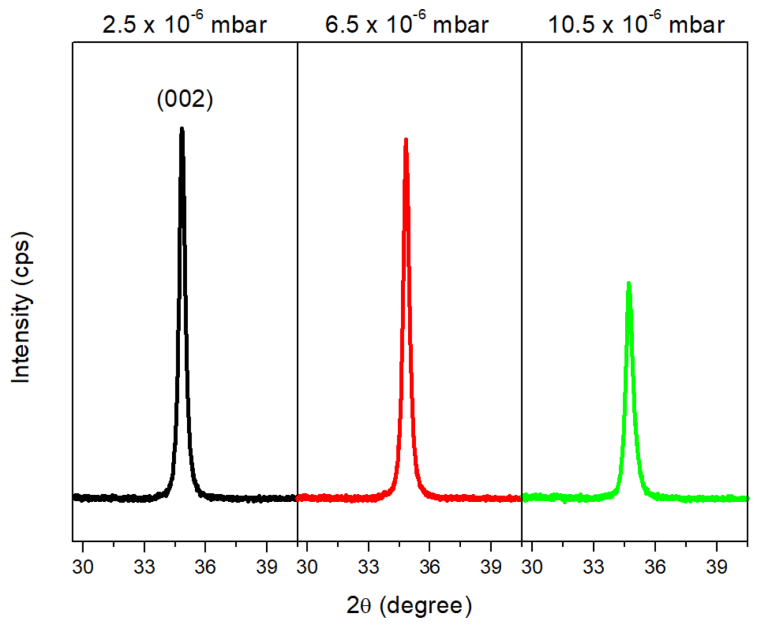

Figure 1. XRD patterns of GZO thin films with various base pressures.

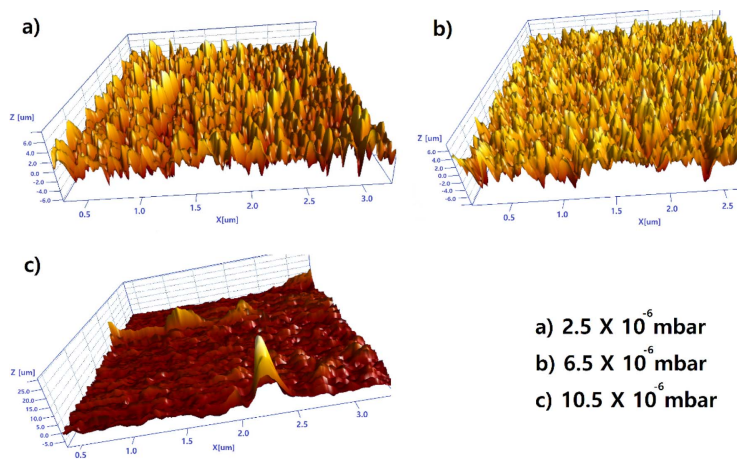

Figure 2. AFM 3D images of GZO thin films with various base pressures.

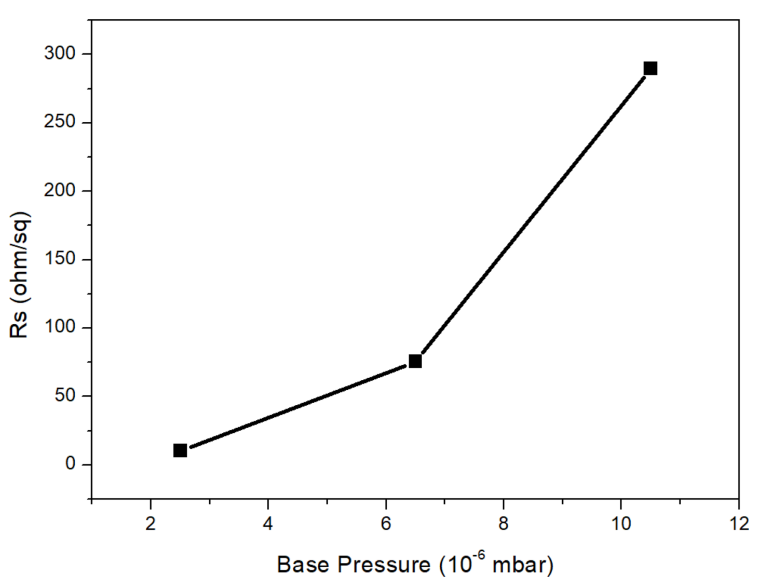

Figure 3. Sheet resistance of GZO thin films with various base pressures.

grain size decreases the residual impurity absorption due to the reduction of grain boundary, resulting in the decrease of sheet resistance [17]. Also, low base pressure minimizes the quantity of residual impurity. It is shown that the lowest sheet resistance is obtained at a base pressure of $2.5 \times 10^{-}$ ${ }^{6}$ mbar.

Figure 4 shows the transmittance spectra of GZO thin films with various base pressure. The average transmittances 


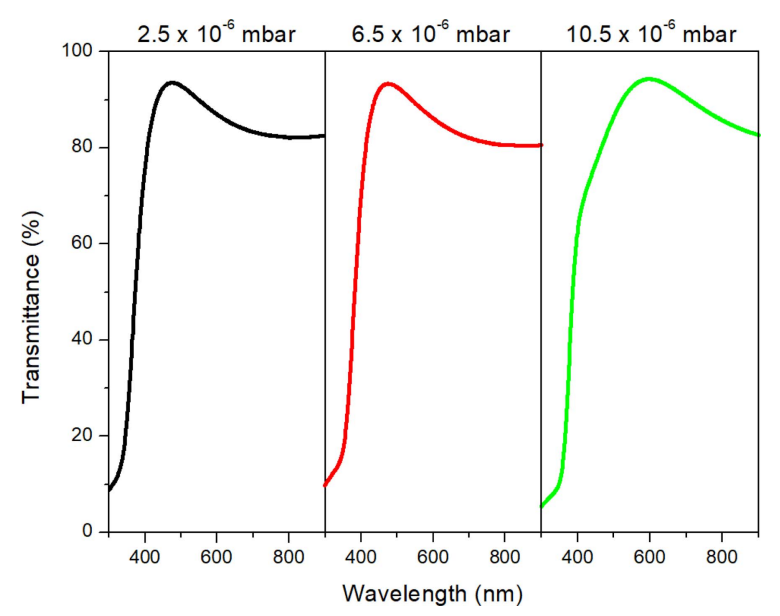

Figure 4. Transmittance spectra of GZO thin films with various base pressures.

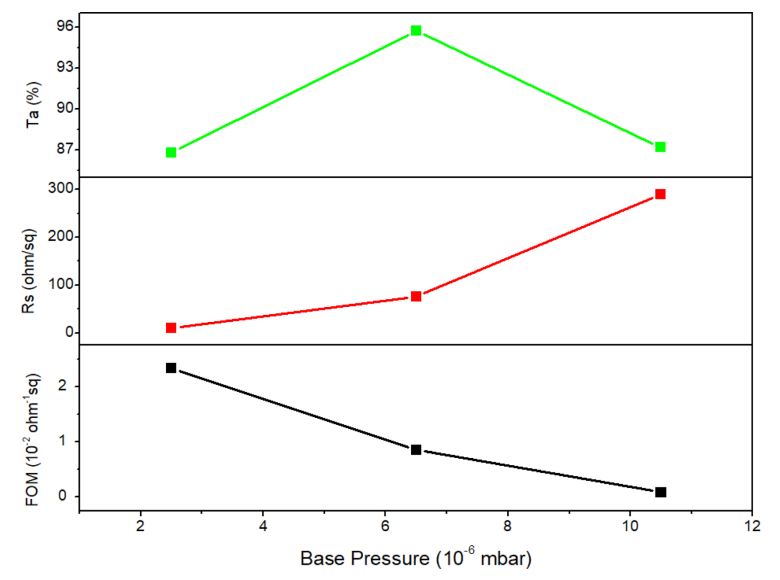

Figure 5. FOM of GZO thin films with various base pressures.

(Ta) of GZO films in visible area $(400 \sim 800 \mathrm{~nm})$ are good values of $85 \sim 87 \%$ but is the independence on base pressure. Also, the light absorption edges around $380 \mathrm{~nm}$ is observed and a blue shift of the absorption edge occurs with decreasing the base pressure, indicating that the band gap is increased. This band gap widening is due to Burstein-Moss effect, since the conduction band becomes significantly filled at high doping concentration and the lowest energy states are blocked which is responsible for the increase in the band gap of the films [18].

Figure 5 represents the figure of merit (FOM) of GZO thin films with various base pressure. The FOM is calculated by Haacke's formula [19]:

$$
\Phi=\frac{T_{a}^{10}}{R S}
$$

where Ta is the average transmittance in the visible range and Rs is the sheet resistance of the film. Using by this formula, the total properties of transparent electrode is derived. As the base pressure decreases, the FOM linearly increases, indicating that the performance of GZO film is improved. At a base pressure of $2.5 \times 10^{-6} \mathrm{mbar}$, the highest FOM of $2.3 \times 10^{-2} \Omega^{-1} \square$ is obtained. In our case, the FOM was strongly dependent on sheet resistance because the change of FOM shows the same trend with that of sheet resistance.

\section{Conclusion}

In this study, the GZO thin films deposited by RF magnetron sputtering and investigated the structural, electrical, and optical properties of GZO thin films with various base pressure. As the base pressure decreases, the crystal quality and sheet resistance is improved. These results are attributed to the reduction of residual impurity. We conclude that the low base pressure plays an important role for obtaining high quality film.

\section{Acknowledgments}

This paper was supported by Wonkwang university in 2017.

\section{References}

[1] H. K. Jeoung and K. M. Lee, J. Ceram. Proc. Res. 17, 281 (2016).

[2] F. Z. Ghomrani, A. Aissat, H. Arbouz, and A. Benkouider, Energy Procedia 74, 491 (2015).

[3] R. H. Hrong, Y. Y. Zeng, W. K. Wang, C. L. Tsai, Y. K. Fu, and W. H. Kuo, Opics Express 25, 32206 (2017).

[4] H. W. Park, K. B. Chung, J. S. Park, S. Ji, K. Song, H. Lim, and M. H. Jang, Ceram. Inter. 41, 1641 (2015).

[5] Y. Hou. Z. Mei, A. Tang, H. Liang, and X. Du, Phys. Status. Solidi A 215, 1800037 (2018).

[6] L. Dintle, P. Luhanga, C. Modiswe, and C. Muiva, Physica E 99, 91 (2018).

[7] H. J. Ko, Y. F. Chen, S. K. Hong, H. Wenisch, T. Yao, D. C. Look, J. Hu, and R. G. Gordon, J. Appl. Phys. 72, 5381 (1992).

[8] H. Makino and H. Shimizu, Appl. Surf. Sci. 439, 839 (2018).

[9] E. Hasabeldaim, O. M. Ntwaeaborwa, R. E. Kroon, E. Coetsee, and H. C. Swart, Optical Mater. 74, 139 (2017).

[10] H. Beh, D. Hiller, and M. Zacharias, Phys. Status. Solidi A 215, 1700880 (2017).

[11] L. H. Wong and Y. S. Lai, Appl. Phys. A 124, 462 (2018).

[12] M. Mickan, U. Helmersson, and D. Horwat, Surf. Coat. Tech. 347, 245 (2018).

[13] S. Inguva, E. McGlynn, and J. P. Mosnier, Thin Solid Films 621, 171 (2017).

[14] K. D. Vargheese, G. M. Bhatnager, and K. L. Kumar, Mat. Sci. Eng. B 83, 242 (2001).

[15] B. D. Cullity, Elements of X-ray Diffraction (Addison-Wesley Reading, MA, 1978) 102.

[16] C. V. Thompson, Annu. Rev. Mater. Sci. 30, 159 (2000).

[17] Y. Igasaki and H. Saito, Thin Solid Films 199, 223 (1991).

[18] J. H. Oha, K. K. Kim, and T. Y. Seong, Appl. Surf. Sci. 257, 2731 (2011).

[19] G. Haacke, J. Appl. Phys. 47, 4086 (1976). 